\title{
IMAGINARIES IN HILBERT SPACES
}

\author{
ITAY BEN-YAACOV AND ALEXANDER BERENSTEIN
}

\begin{abstract}
We characterise imaginaries (up to interdefinability) in Hilbert spaces using a Galois theory for compact unitary groups.
\end{abstract}

\section{INTRODUCTION}

Recall that a Banach space is a normed vector space over $\mathbb{R}$ or $\mathbb{C}$, which is in addition complete with respect to the metric induced by the norm. It is a Hilbert space if the norm is induced by an inner product $\|x\|=\sqrt{\langle x, x\rangle}$ (and then the inner product is unique and can be recovered from the norm). Everything we say here holds both for the class of real Hilbert space and for that of complex ones: in order to avoid repetition, we will assume throughout the paper that all Hilbert spaces are of the same kind.

The model theory of Hilbert and Banach spaces have been studied from many different points of view. Henson defined the notion of positive bounded formulas, approximate satisfaction and other substitutes for first order notions (see [HI02]). In [Iov99] Iovino developed a theory of stability in this framework. He also used some definable equivalence relations and their classes to generalise the Finite Equivalence Relation Theorem and to define strong types. Another approach for this subject comes from the work of the first author on compact abstract theories (see [Ben03]). We approach Hilbert spaces from this second point of view:

- We work in a universal domain $\mathscr{H}$, that is a Hilbert space far bigger than any set of elements or formulas under consideration. It may be convenient to pretend that $\mathscr{H}$ is a proper class. We consider $\mathscr{H}$ as a multi-sorted structure: $B_{n}(\mathscr{H})=\{v \in \mathscr{H}:\|v\| \leq n\}$ is a sort for every $n<\omega$ (the sorts are not disjoint). Unless explicitly said otherwise, we always work in the sort of the unit ball $B_{1}(\mathscr{H})$.

- Our atomic formulas (or predicates) are of the form $P_{n, \lambda<m}, r, s\left(x_{<m}\right)=[r \leq$ $\left.\left\|\sum_{i<m} \lambda_{i} x_{i}\right\| \leq s\right]$, where $m, n<\omega, \lambda_{i} \in \mathbb{C}($ or $\mathbb{R}), r, s \in \mathbb{R}^{+}$and $x_{0}, \ldots, x_{m-1}$ are variables in the sort $B_{n}$. A positive formula is a positive boolean combination of predicates, and a partial type is a set of positive formulas. If fact, we may restrict ourselves to atomic formulas $P_{n, \bar{\lambda}, r, s}(\bar{x})$ where $\lambda_{i} \in \mathbb{Q}[i]$ (or $\mathbb{Q}$ ) and $r, s \in \mathbb{Q}^{+}$, without changing what properties are expressible by a partial type.

Date: August 28, 2003.

The authors would like to thank Frank Wagner and the CIRM for their hospitality during the Simpleton 2002 meeting during which the discussions that led to this paper took place.

The first author would like to thank Ilan Hirshberg for a few important hints.

At the time of the writing of this paper, the first author was a graduate student with the Équipe de logique mathématique of Université Paris VII. 
- With respect to positive formulas, $\mathscr{H}$ is strongly homogeneous and compact. It is therefore a universal domain for a cat.

- An additional property of positive formulas is that they eliminate quantifiers: if $\pi(x, y)$ is a partial type, then there exist partials types $\rho(x)$ and $\tau(x)$ which are equivalent in $\mathscr{H}$ to $\exists y \pi(x, y)$ and $\forall y \pi(x, y)$, respectively. (Do not forget that the variable $y$ is associated to a sort, so the quantification is over that sort, and not over the entire space).

A type-definable set is the collection of realisations in $\mathscr{H}$ of a partial type, possibly with parameters. A type-definable set would usually be of the same cardinality as $\mathscr{H}$ : otherwise, we call it bounded. This is standard model-theoretic terminology, and should not be confused with the meaning of the word "bounded" in metric spaces: after all, since every type-definable set lives in a sort, it is automatically bounded in the metric sense!

An imaginary is an equivalence class $a_{E}$ of a possibly infinite tuple modulo a typedefinable equivalence relation $E$ (in first-order model theory we would call this a $h y$ perimaginary, but the distinction does not seem to make sense for cats). There are some natural applications of imaginaries in Hilbert spaces. In [Ber02] it is proved that finite rank operators on a Hilbert space can be seen as finite tuples from $\mathscr{H}$ modulo a positive formula. It is also shown that compact operators in $\mathscr{H}$ can be interpreted as a countable collection of finite rank operators. The aim of this paper is to characterise arbitrary imaginaries in a Hilbert space.

It should be mentioned that a property is type-definable in the sense defined above if and only if it is definable by a set of positive bounded formulas in the sense of [HI02]. Thus, both logics are semantically equivalent as far as real elements are concerned. The picture changes slightly when we also consider imaginaries. It is well understood how to add imaginary (or hyperimaginary) sorts to a cat, and such a sort enjoys pretty much the same status as the real sort ([Ben03]). One could also treat imaginary sorts with positive bounded formulas in the same way that hyperimaginaries are treated in first-order theories. For this paper, we will use the cat approach.

We assume familiarity with [Ben03] for the general framework of cats. Results related to stability in Hilbert spaces will be quoted from [BB02]. The reader should not be worried about the fact that [BB02] is written in the language of homogeneous model theory, rather than that of cats, since all the notions we are going to need (dividing, canonical bases, etc.) are defined identically (compare for example with [Ben02a]). For general results about stability in Banach space, [Iov99] mentioned above is a good source, although it uses a different approach to the definition of independence than the previous two (following the "French school").

As we said above, $\mathscr{H}$ denotes a universal domain. We use $H, H_{i}$, etc., to denote small Hilbert subspaces, which usually serve as sets of parameters. Lowercase letters $a$, $b, c$, etc., denote imaginaries. If we wish to emphasise that a certain imaginary is the quotient of a tuple $a$ (usually from $B_{1}(\mathscr{H})$ ) by a type-definable equivalence relation $E$, we note it $a_{E}$. Caution: a fixed enumeration of a small Hilbert space $H$ can also be considered as an imaginary and be denoted $a$; as a general rule, we choose notation according to the aspects we wish to emphasise. 


\section{Preliminaries}

1.1. Bounded closure. If $a$ and $b$ are two imaginaries, then we say that $b$ is definable from $a$ if $b$ is the unique realisation of $\operatorname{tp}(b / a)$, and we write $b \in \operatorname{dcl}(a)$ (read: $b$ is in the definable closure of $a$ ). We say that $b$ is bounded over $a$ if the realisations of $\operatorname{tp}(b / a)$ form a bounded set, and we write $b \in \operatorname{bdd}(a)$ (read: $b$ is in the bounded closure of $a$ ). If $a \in \operatorname{dcl}(b)$ and $b \in \operatorname{dcl}(a)$ then $a$ and $b$ are interdefinable. The Hilbert space generated by a tuple $a \subseteq \mathscr{H}$ is precisely $\operatorname{dcl}(a) \cap \mathscr{H}$.

In first order theories, every type-definable equivalence relation is the intersection of type-definable equivalence relations in countably many variables (see [BPW01]). In cats, the same results holds when replacing countable with the cardinality of the language (see [Ben02b]). As the language for Hilbert spaces can be taken to be countable, we get the same result as in first-order theories: every imaginary is interdefinable with a tuple of imaginaries, each of which being the quotient of a countable tuple (call them countable imaginaries). It is a common abuse of notation to identify bdd(b) (which by definition may contain elements from every possible imaginary sort, and is therefore a proper class) with its restriction to countable sorts. The latter is a small set (with respect to $\mathscr{H}$ ), for which we may choose an enumeration, thus rendering $\operatorname{bdd}(a)$ an imaginary. This abuse of notation is justified by the fact that the class bdd $(a)$ and the imaginary $\operatorname{bdd}(a)$ are interdefinable.

Recall that if $H<\mathscr{H}$ is a Hilbert subspace and $v \in \mathscr{H}$, then $P_{H}(v) \in H$ is the orthogonal projection of $v$ on $H$. If $a$ is a tuple in $\mathscr{H}$, then $P_{H}(a)$ is calculated coordinate-wise.

Fact 1.1. Let $a$ and $b$ be (possibly infinite) tuples from $\mathscr{H}$. Let $H_{b}$ be the Hilbert space generated by $b$. Then $t p(a / b)$ is stationary and the canonical base of this type is the Hilbert space $H_{0}$ generated by $P_{H_{b}}(a)$.

Proof. This and other basic results about stability in Hilbert spaces can be found in [BB02].

$\mathrm{QED}_{1.1}$

Lemma 1.2. Let $a_{E}$ be an imaginary. Then there is a Hilbert space $H$ such that $H \subseteq \operatorname{bdd}\left(a_{E}\right)$ and $a_{E} \in \operatorname{dcl}(H)$.

Proof. Let $\left(a_{i}: i \in \omega\right)$ be a Morley sequence in $\operatorname{tp}\left(a / a_{E}\right)$ such that $a_{0}=a$. Let $H_{1}$ be the Hilbert space spanned by $a_{1}$ and $H_{0}$ the one spanned by $P_{H_{1}}(a)$. Then $a \downarrow_{H_{0}} a_{1}$. Since $a_{E} \in \operatorname{dcl}(a) \cap \operatorname{dcl}\left(a_{1}\right)$, then $a_{E} \in \operatorname{bdd}\left(H_{0}\right)$. Since $\operatorname{tp}\left(a / H_{0}\right)$ is stationary, so is $\operatorname{tp}\left(a_{E} / H_{0}\right)$ and in fact $a_{E} \in \operatorname{dcl}\left(H_{0}\right)$. On the other hand, $H_{0}=\operatorname{Cb}\left(\operatorname{tp}\left(a / a_{1}\right)\right)=$ $\mathrm{Cb}\left(\operatorname{lstp}\left(a / a_{E}\right)\right)$, so $H_{0} \subseteq \operatorname{bdd}\left(a_{E}\right)$.

$\mathrm{QED}_{1.2}$

Corollary 1.3. Let a be an imaginary. Then $\operatorname{bdd}(a)$ is (interdefinable with) a unique Hilbert space.

Proof. $\operatorname{bdd}(a)$ is itself an imaginary, so there is a Hilbert space $H$ satisfying $\operatorname{bdd}(a) \subseteq$ $\operatorname{dcl}(H)$ and $H \subseteq \operatorname{bdd}(\operatorname{bdd}(a))=\operatorname{bdd}(a)$. If $b \notin H$ is a real element then $b \notin \operatorname{bdd}(H)$ either, whereby $H$ is unique.

$\mathrm{QED}_{1.3}$

Convention 1.4. As we are only interested in $\operatorname{bdd}(a)$ up to interdefinability, we replace from now on $\operatorname{bdd}(a)$ with the unique Hilbert space interdefinable with it. 
1.2. Compact sets. We try to connect the notions of (bounded and unbounded) typedefinable sets, which take place in the universal domain $\mathscr{H}$, with geometric notions in a relatively small Hilbert space $H<\mathscr{H}$.

Lemma 1.5. A set $X \subseteq B_{1}(H)^{n}$ is closed if and only if it is of the form $X=$ $Y \cap B_{1}(H)^{n}$, where $Y \subseteq B_{1}(\mathscr{H})^{n}$ is a type-definable set (one may say that $X$ is a relatively type-definable subset of $\left.B_{1}(H)^{n}\right)$. Moreover, we may choose $Y$ to be defined over $H$.

Proof. Assume that $X \subseteq B_{1}(H)^{n}$ is closed. For each $a \in B_{1}(H)^{n} \backslash X$, let $r_{a}=d(X, a)$ be the distance between $X$ and $a$ (since $X$ is closed, $r_{a}>0$ ). The partial type saying that $\|x-a\| \geq r_{a}$ for every $a \in B_{1}(H)$ defines $X$ in $B_{1}(H)$ with parameters in $H$. Conversely, the language for Hilbert spaces only defines closed sets.

$\mathrm{QED}_{1.5}$

Corollary 1.6. The map $B_{1}(H)^{n} \rightarrow \mathrm{S}_{n}(H)$ sending $a \mapsto \operatorname{tp}(a / H)$ is a topological embedding.

Proof. This map is clearly injective. By the previous lemma it induces a homeomorphism of $B_{1}(H)^{n}$ with its image.

$\mathrm{QED}_{1.6}$

Corollary 1.7. A subset $K \subseteq B_{1}(H)^{n}$ is compact if and only if it is a (bounded) typedefinable set. (Of course, since it is "small", if it is type-definable it is automatically a bounded one).

Proof. Let $K^{\prime} \subseteq \mathrm{S}_{n}(H)$ be the image of $K$. Then $K$ is type-definable $\Longleftrightarrow K^{\prime}$ is closed $\Longleftrightarrow K^{\prime}$ is compact $\Longleftrightarrow K$ is compact.

$\mathrm{QED}_{1.7}$

Fact 1.8. Let $K \subseteq \mathscr{H}^{n}$ be a bounded type-definable set. Then it has an imaginary canonical parameter.

Proof. This was proved in [BPW01, Lemma 2.18] (for the special case of a hyperimaginary sort in a first order theory, but the same proof holds).

$\mathrm{QED}_{1.8}$

\section{GALOIS THEORY FOR IMAGINARIES}

Fix an imaginary $c$, and let $H=\operatorname{bdd}(c)$ (so of course, $c \in \operatorname{dcl}(H)$ ). Fix an enumeration $H=\left\{h_{i}: i<\lambda\right\}$, and let $Y=y_{<\lambda}$ where each variable $y_{i}$ is in the sort corresponding to the smallest ball containing $h_{i}$. Let $p(x, Y)=\operatorname{tp}(c, H)$, and let $G=\left\{q(Y, Z) \in \mathrm{S}_{Y, Z}(\varnothing): \exists x p(x, Y) \wedge p(x, Z) \wedge q(Y, Z)\right\}$. Assume that $q(Y, Z) \in G$. Then all it says about $Y$ is that it satisfies $\operatorname{tp}(H)$, and in particular $q(H, Z)$ is consistent. However, if $\vDash q\left(H, H^{\prime}\right)$, then $H^{\prime}=\left\{h_{i}^{\prime}: i<\lambda\right\}$ is also an enumeration of $\operatorname{bdd}(c)$, so it is just another enumeration of $H$. Therefore, for every $i<\lambda$ there is a unique $q[i]<\lambda$ such that $h_{q[i]}=h_{i}^{\prime}$, or equivalently $q(Y, Z) \vdash y_{q[i]}=z_{i}$. Thus $q$ determines a map $g^{q} \in \operatorname{Aut}(H / c)$ defined by $g^{q}\left(h_{i}\right)=h_{q[i]}$. Conversely, if $g \in \operatorname{Aut}(H / c)$ and $q^{g}=\operatorname{tp}(H, g(H))$, then $q^{g} \in G$ and $g=g^{q^{g}}$. We conclude that $G$ can be identified with the group $\operatorname{Aut}(H / c)$. However, it is also a compact Hausdorff space as a closed subset of $\mathrm{S}_{Y, Z}(\varnothing)$, and we would like to show that the two structures agree.

For $q, r \in G$, define $(r \circ q)(Y, Z)=\exists W r(Y, W) \wedge q(W, Z)$. Then for $i<\lambda$ we have $q(W, Z) \vdash w_{q[i]}=z_{i}$ and $r(Y, W) \vdash y_{r[i]}=w_{i}$, whereby $(r \circ q)(Y, Z) \vdash y_{r[q[i]]}=z_{i}$. This shows that $g^{r \circ q}=g^{r} \circ g^{q}$. For $F \subseteq G$, we have $\left\{(r, q) \in G^{2}: r \circ q \in F\right\}=\theta\left(\psi^{-1}(F)\right) \cap G^{2}$, where $\psi: \mathrm{S}_{Y, W, Z}(\varnothing) \rightarrow \mathrm{S}_{Y, Z}(\varnothing)$ and $\theta: \mathrm{S}_{Y, W, Z}(\varnothing) \rightarrow \mathrm{S}_{Y, W}(\varnothing) \times \mathrm{S}_{W, Z}(\varnothing)$ are the obvious 
restriction maps, which are continuous. If $F$ is closed in $G$ then it is closed in $\mathrm{S}_{Y, Z}(\varnothing)$ as well, so $\psi^{-1}(F)$ is closed and therefore compact. Then $\theta\left(\psi^{-1}(F)\right)$ is compact, and therefore closed, and we conclude that $\theta\left(\psi^{-1}(F)\right) \cap G^{2}$ is closed, and that composition is continuous. Given the definition of composition it is clear that the inverse operation in $G$ is defined by $q(Y, Z) \mapsto q(Z, Y)$, which is clearly continuous in $\mathrm{S}_{Y, Z}(\varnothing)$.

Thus $G$ is a compact topological group.

In fact, the argument given above only uses the fact that we work in a Hausdorff cat (see [Ben03]), and that we consider a group of the form $G=\operatorname{Aut}(\operatorname{bdd}(c) / c)$ for some imaginary $c$. We call $G$ the absolute Galois group of $c$, and write $G=\operatorname{Gal}(c)$ and the topology the Galois topology (one may compare with [LP01]).

We have shown that $G$ carries a very natural model-theoretic topology. However, we also have an inclusion $G<U(H)$, where $U(H)$ is the unitary group of $H$. For our needs, the natural topology on $U(H)$ is the strong operator topology, namely that induced by the Tychonoff topology on $H^{H}$ (where $H$ is taken with the ordinary norm topology). This topology can also characterised by convergence: a network $\left(g_{\alpha}\right)$ converges strongly to $g$ if and only if $g_{\alpha}(a) \rightarrow g(a)$ for every $a \in H$.

Lemma 2.1. The Galois topology on $G$ coincides with the strong topology.

Proof. Let $g \in G, a \in H$ and $r>0$, and let $V=\left\{g^{\prime} \in G:\left\|g(a)-g^{\prime}(a)\right\|<r\right\}$. We want to show that $V$ is a neighbourhood of $g$ in the Galois topology. Assume that $a=h_{i}$ and $g(a)=h_{j}$. Then the set $V$ is the set of all types $q(Y, Z) \in G$ satisfying $\left\|y_{j}-z_{i}\right\|<r$, which is open. This shows that the strong topology is weaker than the Galois topology. Since the Galois topology is compact, and the strong topology is Hausdorff, they must coincide.

$\mathrm{QED}_{2.1}$

And the converse is:

Lemma 2.2. Let $H$ be any Hilbert space, and $G<U(H)$ be any compact subgroup in the strong topology. Then there is an imaginary $c$ such that $H=\operatorname{bdd}(c)$ and $G=\operatorname{Gal}(c)$.

Proof. Let $\left(e_{i}: i<\lambda\right)$ be an orthonormal basis for $H$, and set $\mathcal{I}=[\lambda]^{<\omega}$. For every $I \in \mathcal{I}$, the orbit of $\left(e_{i}: i \in I\right)$ under the action of $G$ is a compact subset of $B_{1}(H)^{I}$, which will be denoted by $K_{I}$. By Corollary $1.7 K_{I}$ is type-definable, and by Fact 1.8 it has a canonical parameter $c_{I}$. Let $c=\left(c_{I}: I \in \mathcal{I}\right)$.

Clearly, $\operatorname{bdd}(c) \subseteq H$, but we also have $e_{i} \in K_{\{i\}} \subseteq \operatorname{bdd}\left(c_{\{i\}}\right)$ for every $i<\lambda$, whereby we have equality $\operatorname{bdd}(c)=H$. It is also clear that $G$ fixes every $c_{I}$, and therefore it fixes $c$.

Conversely, assume that $g \in U(H)$ fixes $c$. Then $g$ fixes $c_{I}$ for every $I \in \mathcal{I}$, which means that there is some $g_{I} \in G$ such that $g_{I}\left(e_{i}\right)=g\left(e_{i}\right)$ for every $i \in I$. Let $a=\sum_{i<\lambda} \alpha_{i} e_{i} \in H$ be any element. Then for every $r>0$ there is $I \in \mathcal{I}$ such that $\left\|\sum_{i \notin I} \alpha_{i} e_{i}\right\|<r / 2$, whereby $\left\|g(a)-g_{J}(a)\right\|<r$ for every $J \in \mathcal{I}$ containing $I$. This shows that $g_{I}(a) \rightarrow g(a)$ as a network in $H$ for every $a \in H$, whereby $g_{I} \rightarrow g$ in $U(H)$ (with the strong topology). Since $G$ is closed in $U(H)$ we obtain $g \in G$. $\quad \mathrm{QED}_{2.2}$

We conclude:

Theorem 2.3. Given a Hilbert space $H$, there is a Galois correspondence between imaginaries $c$ such that $H=\operatorname{bdd}(c)$ (up to interdefinability) and compact subgroups of 
$U(H)$ in the strong topology, given by:

$$
\begin{aligned}
c & \mapsto \operatorname{Gal}(c) \\
G & \mapsto H^{G}=\{c \in \operatorname{dcl}(H): \forall g \in G[g(c)=c]\}
\end{aligned}
$$

\section{Elimination to Finitary imaginaries}

We already know that every imaginary is interdefinable with a set of countable ones. Using the previous results and a theorem on representations of compact groups we improve "countable" to "finite":

Definition 3.1. An imaginary is finitary if it is (interdefinable with an imaginary) of the form $a_{E}$ where $a$ is a finite tuple.

Lemma 3.2. An imaginary $a$ is finitary if and only if $H=\operatorname{bdd}(a)$ is finitedimensional.

Proof. Clear.

$\mathrm{QED}_{3.2}$

Recall that if $\left(H_{\alpha}: \alpha \in I\right)$ are Hilbert spaces, then their Hilbert direct sum $\bigoplus_{\alpha} H_{\alpha}$ is defined as the set of all tuples $\left(v_{\alpha}\right) \in \prod_{\alpha} H_{\alpha}$ satisfying $\sum_{\alpha}\left\|v_{\alpha}\right\|^{2}<\infty$. It has an obvious structure of a vector space; equipped with the inner product $\langle\bar{v}, \bar{u}\rangle=$ $\sum_{\alpha}\left\langle v_{\alpha}, u_{\alpha}\right\rangle$ it is a Hilbert space, and in fact it is the co-product of $\left(H_{\alpha}\right)$ in the category of Hilbert spaces. We now introduce a tool from analysis:

Fact 3.3. Let $G<U(H)$ be compact in the strong topology. Then $H$ can be expressed as a Hilbert direct sum $H=\bigoplus_{\alpha} H_{\alpha}$, where each $H_{\alpha}$ is finite-dimensional and $G$ invariant.

Proof. See the fundamental theorem on unitary modules and Corollary 2.25 in [HM98]. $\mathrm{QED}_{3.3}$

Proposition 3.4. Every imaginary is interdefinable with a tuple of finitary imaginaries.

Proof. We refine the proof of Lemma 2.2, by taking the basis $\left(e_{i}: i<\lambda\right)$ to be a union of orthonormal bases for the $H_{\alpha}$. Then for every $I \in \mathcal{I}=[\lambda]^{<\omega}$, the tuple $e_{\in I}$ belongs to the sum of a finite number of such $H_{\alpha}$, which is also a finite-dimensional $G$-invariant space. Thus every $K_{I}$ is contained in a finite-dimensional space, and $c_{I}$ is finitary. Applying this to $\operatorname{Gal}(c)$ we obtain that $c$ is interdefinable with $\left(c_{I}: I \in \mathcal{I}\right) . \quad \operatorname{QED}_{3.4}$

The very last step is to understand finitary imaginaries:

Definition 3.5. For $n<\omega$, the group of unitary matrices $U(n)$ acts in an obvious manner on $\mathscr{H}^{n}$. Let $G<U(n)$ and $\bar{e}, \bar{f} \in \mathscr{H}^{n}$. Then we say that $\bar{e} E_{G} \bar{f}$ if $\bar{e}$ and $\bar{f}$ are orthonormal tuples and $g(\bar{e})=\bar{f}$ for some $g \in G$.

Lemma 3.6. Assume that $c$ is finitary, and let $\left(e_{i}: i<n\right)$ be an orthonormal basis for $H=\operatorname{bdd}(c)$. Let $G=\operatorname{Gal}(c)<U(H)=U(n)$, where we identify $U(H)$ with $U(n)$ through the basis $\left(e_{i}: i<n\right)$. Then $E_{G}$ is type-definable, and $c$ is interdefinable with $\bar{e}_{E_{G}}$. 
Proof. Let $p(x, \bar{y})=\operatorname{tp}(c, \bar{e})$ and $E(\bar{y}, \bar{z})=\exists x p(x, \bar{y}) \wedge p(x, \bar{z})$. We wish to show that for every $\bar{e}^{\prime}, \bar{f} \in \mathscr{H}^{n}: \bar{e}^{\prime} E \bar{f} \Longleftrightarrow \bar{e}^{\prime} E_{G} \bar{f}$. Since both conditions are automorphisminvariant, and either would imply that $\bar{e}^{\prime}$ is an orthonormal tuple, we may assume that $\bar{e}=\bar{e}^{\prime}$. Then $\bar{e} E \bar{f}$ if and only if there is an automorphism of $H$ fixing $c$ and sending $\bar{e}$ to $\bar{f}$, and this is equivalent to $\bar{e} E_{G} \bar{f}$. Thus $c$ is interdefinable with $\bar{e}_{E}$, and $E_{G}=E$ is type-definable.

$\mathrm{QED}_{3.6}$

Corollary 3.7. $E_{G}$ is type-definable for every closed $G<U(n)$.

Proof. By Theorem 2.3, every closed $G<U(n)$ occurs as a Galois group. $\quad \operatorname{QED}_{3.7}$

We conclude:

Theorem 3.8. Every imaginary is interdefinable with a tuple of imaginaries of the form $\bar{e}_{E_{G}}$, where each $G<U\left(n_{\bar{e}}\right)$ is closed and $\bar{e}$ is an orthonormal tuple of length $n_{\bar{e}}$. In other words, Hilbert spaces eliminate imaginaries to quotients of finite orthonormal tuples by closed unitary groups.

Remark 3.9. This is reminiscent of the situation in the (first order) theory of the infinite set. There, if $a$ is an imaginary (since we deal with a first order theory, we revert to the definition of an imaginary as the quotient of a finite tuple by an equivalence relation definable by a single formula), then $\operatorname{acl}(a)$ is (interdefinable with) a finite tuple $e_{0}, \ldots, e_{n-1}$, and then $a$ is interdefinable with $\bar{e} / E_{G}$ where $G$ is a permutation group of $n$ elements, acting naturally on the tuple $\bar{e}$. Hyperimaginaries can be eliminated in favour of imaginaries by general first order stability theory.

The only difference from the Hilbert space case is the replacement of "finite tuple" with "finite dimensional" or "finite basis", and of the symmetric group $S_{n}$ with $U(n)$.

\section{REFERENCES}

[BB02] Alexander Berenstein and Steven Buechler, Homogeneous expansions of Hilbert spaces, preprint, 2002.

[Ben02a] Itay Ben-Yaacov, Simplicity in compact abstract theories, preprint, 2002.

[Ben02b] Itay Ben-Yaacov, Thickness, and a categoric view of type-space functors, preprint, 2002.

[Ben03] Itay Ben-Yaacov, Positive model theory and compact abstract theories, Journal of Mathematical Logic 3 (2003), no. 1, 85-118.

[Ber02] Alexander Berenstein, Dividing in the $C^{*}$-algebra of compact operators, preprint, 2002.

[BPW01] Steven Buechler, Anand Pillay, and Frank O. Wagner, Supersimple theories, Journal of the American Mathematical Society 14 (2001), 109-124.

[HI02] C. Ward Henson and José Iovino, Ultraproducts in Analysis, Analysis and Logic (Catherine Finet and Christian Michaux, eds.), London Mathematical Society Lecture Notes Series, no. 262, Campridge University Press, 2002.

[HM98] Karl H. Hoffmann and Sidney A. Morris, The structure of compact groups, de Gruyter Studies in Mathematics, no. 25, Walter de Gruyter, 1998.

[Iov99] José Iovino, Stable Banach spaces and Banach space structures, I and II, Models, Algebras, and Proofs (Bogotá, 1995), Lectures Notes in Pure and Appl. Math., no. 203, Dekker, New York, 1999, pp. 77-117.

[LP01] Daniel Lascar and Anand Pillay, Hyperimaginaries and automorphism groups, Journal of Symbolic Logic 66 (2001), 127-143. 
Itay Ben-Yaacov, Massachusetts Institute of Technology, Department of Mathematics, 77 Massachusetts Avenue, Room 2-101, Cambridge, MA 02139-4307, USA

E-mail address: pezz@math.mit.edu

$U R L:$ http://www.logique.jussieu.fr/www.pezz

Alexander Berenstein, University of Illinois at Urbana-Champaign, 1409 West Green Street, Urbana, IL 61801-2975, USA

E-mail address: aberenst@math.uiuc.edu 